WP 28_13

Zhongxian Men

University of Waterloo, Canada

Adam W. Kolkiewicz

University of Waterloo, Canada

Tony S. Wirjanto

University of Waterloo, Canada

The Rimini Centre for Economic Analysis (RCEA), Italy

\title{
BAYESIAN INFERENCE OF ASYMMETRIC STOCHASTIC CONDITIONAL DURATION MODELS
}

Copyright belongs to the author. Small sections of the text, not exceeding three paragraphs, can be used provided proper acknowledgement is given.

The Rimini Centre for Economic Analysis (RCEA) was established in March 2007. RCEA is a private, nonprofit organization dedicated to independent research in Applied and Theoretical Economics and related fields. RCEA organizes seminars and workshops, sponsors a general interest journal The Review of Economic Analysis, and organizes a biennial conference: The Rimini Conference in Economics and Finance (RCEF) . The RCEA has a Canadian branch: The Rimini Centre for Economic Analysis in Canada (RCEACanada). Scientific work contributed by the RCEA Scholars is published in the RCEA Working Papers and Professional Report series.

The views expressed in this paper are those of the authors. No responsibility for them should be attributed to the Rimini Centre for Economic Analysis. 


\title{
Bayesian Inference of Asymmetric Stochastic Conditional Duration Models
}

\author{
Zhongxian Men ${ }^{\mathrm{a}}$, Adam W. Kolkiewicz ${ }^{\mathrm{a}}$, Tony S. Wirjanto ${ }^{\mathrm{a}, *}$ \\ ${ }^{a}$ Department of Statistics \& Actuarial Science, University of Waterloo, Waterloo, \\ Ontario, Canada N2L $3 G 1$
}

\begin{abstract}
This paper extends stochastic conditional duration (SCD) models for financial transaction data to allow for correlation between error processes or innovations of observed duration process and latent log duration process. Novel algorithms of Markov Chain Monte Carlo (MCMC) are developed to fit the resulting SCD models under various distributional assumptions about the innovation of the measurement equation. Unlike the estimation methods commonly used to estimate the SCD models in the literature, we work with the original specification of the model, without subjecting the observation equation to a logarithmic transformation. Results of simulation studies suggest that our proposed models and corresponding estimation methodology perform quite well. We also apply an auxiliary particle filter technique to construct one-step-ahead in-sample and out-of-sample duration forecasts of the fitted models. Applications to the IBM transaction data allows comparison of our models and methods to those existing in the literature.

Keywords: Stochastic Duration; Bayesian Inference; Markov Chain Monte Carlo; Leverage Effect; Acceptance-rejection; Slice Sampler.
\end{abstract}

\section{Introduction}

There has been an increasing interest in the intraday financial transaction data among researchers in empirical finance at least for two reasons: (i) The timing of transactions carries useful information about the microstructure of

\footnotetext{
${ }^{*}$ Corresponding author

Email address: twirjant@uwaterloo.ca (Tony S. Wirjanto)
}

February 19, 2013 
financial markets, and (ii) Increasing access to high frequency transaction data due partly to a rapid development in electronic trading and computer technology. Various statistical models and methods have been developed to characterize the properties of these high frequency data. This paper extends Stochastic Conditional Duration (SCD) models studied by Bauwens and Veredas (2004) to allow for correlation between the two error process or innovations of the model. In addition, to estimate parameters of the models and log durations, novel Markov Chain Monte Carlo (MCMC) algorithms are developed in which the latent states (the log durations) are estimated as a by-product of the estimation process.

A salient feature of financial duration data is that they are irregularly spaced. Traditional models, such as the autoregressive conditional heteroskedasticity (ARCH) model of Engle (1982), the generalized autoregressive conditional heteroskedasticity (GARCH) models of Bollerslev (1986), and the stochastic volatility (SV) model of Taylor (1986) are not suitable for this type of data as they require the data being analyzed to be regularly spaced. To work with the time series of financial durations, Engle and Russel (1998) propose an autoregressive conditional duration (ACD) model similar to the $\mathrm{ARCH} / \mathrm{GARCH}$ models. The only difference between them is that the innovation of the ACD model has a positive support region. One of the advantages of the ACD model is that maximum likelihood (ML)-based estimation and inference for the model's parameters is relatively easy and straightforward to carry out.

Due to its similarity to the GARCH model, the ACD model shares many properties with the GARCH model. One notable feature of the ACD model is that the conditional duration is a deterministic function of the previous durations and innovations. This is felt to unduly restrict the process of the expected conditional durations. For this reason, Bauwens and Veredas (2004) generalize the ACD model to allow the logarithm of the expected conditional duration to follow a first-order stationary autoregressive $(\mathrm{AR}(1))$ process with a univariate normal innovation, and innovation of the observation equation to follow either a Gamma or a Weibull distribution with a scale parameter set to unity. It is further assumed that the two innovations are mutually independent. As the analytical likelihood function of the data under the SCD model is difficult to obtain, both analytical and simulation-based estimation methods have been applied to the log transformation of the SCD model. Assuming no correlation between the innovations of the transformed model, Bauwens and Veredas (2004) estimate the model's parameters via a quasi-maximum 
likelihood(QML) method with the Kalman filter. For this model, Strickland, Forbes, and Martin (2006) propose a Bayesian MCMC method where the latent states are augmented as parameters and estimated by the MetropolisHastings (MH) method. Knight and Ning (2008) use generalized method of moments (GMM) to estimate the parameters of the SCD model, where the moments are calculated from the empirical characteristic function (ECF). Bauwens and Galli (2009) develop an efficient importance sampling method for estimating the parameters of the SCD models. Allowing for correlation (which is interpreted as a leverage effect) between the transformed equation and the latent process, Feng, Jiang, and Song (2004) propose a ML method, which was originally proposed by Durbin and Koopman (2004), to estimate the parameters of the transformed SCD model. More recently, Xu, Knight, and Wirjanto (2011) relax the distributional assumption of the two innovations of the transformed SCD model even further by using a two-component mixture of bivariate normal distributions to approximate the joint distribution of the two innovations. This mixture distribution allows the transformed model to be more flexible in its ability to accommodate the complex bivariate distributions of the two innovations. A continuous ECF approach is developed by the authors to estimate the parameters of the transformed SCD model based on transaction data from IBM and Boeing.

It is important to point out that the correlation between the innovations in the log transformed model may not capture the leverage effect properly as the latter is, strictly speaking, intended to measure the correlation between the innovations in the original specification of the SCD model. Motivated by this argument, we propose to estimate the leverage effect, which measures the correlation between the two innovations in the original, untransformed SCD model. We expect that this quantity better characterizes the dependence structure between the duration dynamics and the log duration process in the data. Indeed we find that, using our proposed model, the estimated correlation coefficient from duration data is always found to be negative. For this reason, the model is called an asymmetric SCD (ASCD) model. This represents the first contribution of our paper. Our second contribution is to develop novel MCMC algorithms to estimate the parameters and log durations of the ASCD model, where the innovation of the measurement equation is assumed to follow either a Weibull, a Gamma or an Exponential distribution. In the proposed MCMC algorithms, the state random variables are augmented as parameters and simulated one at a time through a MH method, where the proposal distribution is sampled via a slice sampler proposed in 
Edwards and Sokal (1998) and Neal (2003). The third contribution of this paper is to construct one-step-ahead in-sample and out-of-sample duration forecasts for the ASCD model by applying the auxiliary particle filter (APF) introduced by Pitt and Shephard (1999b). Duration forecasting is empirically important in terms of portfolio allocation or risk measurement.

The remaining parts of the paper are organized as follows. Section 2 reviews the SCD model and existing estimation approaches. The proposed ASCD model and developed MCMC methods for the estimation of the parameters and latent states of the SCD model are presented in Section 3. Goodness-of-fit test and model assessment are discussed in this section as well. Simulation studies are conducted in Section 4. Section 5 presents an application of our proposed models and methods to the IBM transaction data, where the Bayesian inference of the parameters of the model are compared to those from the other competing methods. Conclusions are provided in Section 6.

\section{The Stochastic Conditional Duration model}

In this section, we briefly review the SCD model and the existing estimation approaches.

The model has been proposed by Bauwens and Veredas (2004) to describe time series of stochastic durations. If we denote by $y_{t}$ the observed duration at time $t, t \leq T$, then the authors propose to represent $y_{t}$ as a product of a lognormal random variable $h_{t}$ and a positive random variable $\epsilon_{t}$. More specifically, the SCD model is given by

$$
\begin{array}{rlrl}
y_{t} & =\exp \left(h_{t}\right) \epsilon_{t}, & t=1, \ldots, T, \\
h_{t+1} & =\mu+\phi\left(h_{t}-\mu\right)+\sigma \eta_{t+1}, & t=1, \ldots, T-1, & \\
h_{1} & \sim \mathcal{N}\left(\mu, \sigma^{2} /\left(1-\phi^{2}\right)\right), &
\end{array}
$$

where $\eta_{2}, \eta_{3}, \ldots$, are independent random variables such that $\eta_{t} \sim \mathcal{N}(0,1)$, with $\mathcal{N}(a, b)$ denoting a normal distribution with mean $a$ and variance $b$. It is assumed that $\epsilon_{1}, \epsilon_{2}, \ldots$, are independent with a common distribution that has a positive support, and the error processes $\epsilon_{t}$ and $\eta_{t+1}$ are independent. To ensure weak stationarity of the latent $\operatorname{AR}(1)$ process, we assume that $|\phi|<1$. Bauwens and Veredas (2004) also assume that $\epsilon_{t}$ follows either a $\operatorname{Weibull}(\gamma, 1)$ or a $\operatorname{Gamma}(v, 1)$ distribution, with the densities given by

$$
f(x)=v x^{v-1} \exp \left(-x^{v}\right)
$$


and

$$
f(x)=\frac{1}{\Gamma(\gamma)} x^{\gamma-1} \exp (-x)
$$

respectively. Both distributions are allowed to have unknown shape parameters, but the scale parameters are set equal to 1 . These distributions reduce to an exponential distribution when $v$ and $\gamma$ are equal to 1.

As the observation equation (1) is highly non-linear, the likelihood function does not have a closed form expression, and hence integrating out the $T$ latent random variables is a challenging problem. In most prior studies, a $\log$ transformation is therefore performed on the observation equation (1) to obtain:

$$
\log \left(y_{t}\right)=h_{t}+e_{t}, \quad t=1, \ldots, T,
$$

where $e_{t}=\log \left(\epsilon_{t}\right)$ is the transformed error process and the latent $\operatorname{AR}(1)$ process describing $h_{t}$ is left unchanged. Then the variables $e_{t}$ have either a Log-Weibull or a Log-Gamma distribution, with the densities respectively given by

$$
f(x)=v \exp \left(v x-e^{v}\right)
$$

and

$$
f(x)=\frac{1}{\Gamma(\gamma)} \exp \left(\gamma x-e^{\gamma x}\right)
$$

To estimate the parameters of this transformed SCD model, several methods have been proposed in the literature. As we discussed earlier, all the estimation methods work with the transformed SCD model defined by (6), (2), and (3). It is worth noting that although the log-transformation (6) is a one-to-one mapping, the correlation coefficient in the model with the leverage effect introduced between the transformed innovation processes is not the same as the correlation between the two original innovation processes. Therefore results concerning the dependence structure of the innovation processes obtained by fitting a linear regression model to the log-transformed data should not be automatically extended to the original data. Some pitfalls of estimating correlations from transformed data are discussed, among others, by Sandmann and Koopman (1998) in the context of the Monte Carlo maximum likelihood method for stochastic volatility models. An analysis presented in this paper confirms the need for a cautious interpretation of estimation results based on transformed data. In particular, while Feng, Jiang, and Song (2004) and Xu, Knight, and Wirjanto (2011) find that there is a positive correlation between the two transformed innovations, in this paper 
we find that the innovations of the original SCD model are negatively correlated. This leads to a seemingly more plausible interpretation of the captured correlation as a leverage effect.

\section{Asymmetric SCD model}

\subsection{Model and its properties}

Here we introduce a model for financial duration time series that does not subject the data to a logarithmic transformation. Our approach is based on the model specification given by equations (1)-(3). However, instead of characterizing the leverage effect as a correlation between $e_{t}$ and $\eta_{t+1}$, we put the innovation $\epsilon_{t}$ into the latent $\mathrm{AR}(1)$ process, so that the latter is driven jointly by $\epsilon_{t}$ and $\eta_{t+1}$. Thus the proposed model, which we call an ASCD model, is specified as:

$$
\begin{aligned}
y_{t} & =\exp \left(h_{t}\right) \epsilon_{t}, \\
h_{t+1} & =\mu+\phi\left(h_{t}-\mu\right)+\psi \epsilon_{t}+\sigma \eta_{t+1}, \\
h_{1} & \sim \mathcal{N}\left(\mu, \sigma^{2} /\left(1-\phi^{2}\right)\right)
\end{aligned}
$$

The additional term $\psi \epsilon_{t}$ in (10) introduces a dependency structure between the innovation processes, and the parameter $\psi$ is expected to capture the correlation between $y_{t}$ and $h_{t+1}$.

To present some properties of the ASCD model, let us define $g_{p}=E\left(\epsilon_{t}^{p}\right)$, for $p=1,2, \kappa=g_{2} / g_{1}^{2}$ and $H_{t}=\exp \left(h_{t}\right), t=1, \ldots, T$. The next two propositions describe moments of $y_{t}$ and $H_{t}$. In Appendix A we prove Proposition 1 , and since the proof of Proposition 2 is similar we omit it.

Proposition 1. Assume that in the model (9)-(11) we have $\epsilon_{t} \sim \operatorname{Gamma}(\gamma, 1)$. Given that $\left|\psi \phi^{i}\right|<1 / 2$, for $i=1,2, \ldots$, the durations $y_{t}$ and the variables $H_{t}$ have the following moments:

$$
\begin{aligned}
& \mu_{H}=e^{\mu+1 / 2 \sigma^{2} /\left(1-\phi^{2}\right)}\left[\prod_{i=0}^{\infty}\left(1-\psi \phi^{i}\right)\right]^{-\gamma}, \\
& \mu_{y}=g_{1} \mu_{H}, \\
& \sigma_{H}^{2}=\mu_{H}^{2}\left(e^{\sigma^{2} /\left(1-\phi^{2}\right)} \prod_{i=0}^{\infty} \frac{\left(1-\psi \phi^{i}\right)^{2 \gamma}}{\left(1-2 \psi \phi^{i}\right)^{\gamma}}-1\right), \\
& \sigma_{y}^{2}=\mu_{y}^{2}\left(\kappa e^{\sigma^{2} /\left(1-\phi^{2}\right)} \prod_{i=0}^{\infty} \frac{\left(1-\psi \phi^{i}\right)^{2 \gamma}}{\left(1-2 \psi \phi^{i}\right)^{\gamma}}-1\right) .
\end{aligned}
$$


In typical applications of the SCD model presented in the literature, $\phi$ is always observed to be positive, so a sufficient condition for the first two moments of the durations and latent variables to be finite is that $\psi<0$. In our applications of the model, we have found that this condition was always satisfied. When $\epsilon_{t}$ follows an exponential distribution, we have the following result.

Proposition 2. Assume that in the model (9)-(11) we have $\epsilon_{t} \sim \operatorname{Exp}(\lambda)$. Given that $\left|\psi \lambda \phi^{i}\right|<1 / 2$, for $i=1,2, \ldots$, the durations $y_{t}$ and the variables $H_{t}$ have the following moments:

$$
\begin{aligned}
\mu_{H} & =e^{\mu+1 / 2 \sigma^{2} /\left(1-\phi^{2}\right)} \prod_{i=0}^{\infty} \frac{1}{1-\psi \lambda \phi^{i}}, \\
\mu_{y} & =g_{1} \mu_{H}, \\
\sigma_{H}^{2} & =\mu_{H}^{2}\left(e^{\sigma^{2} /\left(1-\phi^{2}\right)} \prod_{i=0}^{\infty} \frac{\left(1-\psi \lambda \phi^{i}\right)^{2}}{1-2 \psi \lambda \phi^{i}}-1\right), \\
\sigma_{y}^{2} & =\mu_{y}^{2}\left(\kappa e^{\sigma^{2} /\left(1-\phi^{2}\right)} \prod_{i=0}^{\infty} \frac{\left(1-\psi \lambda \phi^{i}\right)^{2}}{1-2 \psi \lambda \phi^{i}}-1\right) .
\end{aligned}
$$

The next result provides sufficient conditions for stationarity and ergodicity of the duration process and its logarithm $d_{t}:=\log \left(y_{t}\right), t=1,2, \ldots$, which satisfies

$$
d_{t}=h_{t}+e_{t},
$$

where $e_{t}=\log \left(\epsilon_{t}\right)$. A proof of this result is presented in Appendix A.

Proposition 3. The duration process $\left\{y_{t}\right\}$ and its logarithm $\left\{d_{t}\right\}$ are weakly stationary and geometrically ergodic if $|\phi|<1$.

\subsection{Estimation}

For the convenience of developing the MCMC estimation algorithm, we rewrite the model as follows:

$$
\begin{aligned}
y_{t} & =\exp \left(h_{t}\right) \epsilon_{t}, \\
h_{t+1} & =\mu+\phi\left(h_{t}-\mu\right)+\psi y_{t} \exp \left(-h_{t}\right)+\sigma \eta_{t+1}, \\
h_{1} & \sim \mathcal{N}\left(\mu, \sigma^{2} /\left(1-\phi^{2}\right)\right),
\end{aligned}
$$


Define $\theta_{G}=(\mu, \phi, \psi, \sigma, \gamma), \theta_{W}=(\mu, \phi, \psi, \sigma, v)$, and $\theta_{E}=(\mu, \phi, \psi, \sigma)$ as the three vectors of parameters for the ASCD model when the observation equation is based either on a $\operatorname{Gamma}(\gamma, 1)$, a Weibull $(v, 1)$ or an Exponential(1) distribution. We will call the corresponding ASCD models ASCD-W, ASCD-G and ASCD-E, respectively. Let $\mathbf{y}=\left(y_{1}, \ldots, y_{T}\right)$ be the set of observed durations and $\mathbf{h}=\left(h_{1}, \ldots, h_{T}\right)$ be the set of the corresponding latent states.

In the implementation of the MCMC algorithm, $\mathbf{h}$ is augmented as a vector of parameters and estimated as a by-product of the estimation process. Let $f($.$) be a generic density function. From now on, we use \theta$ to represent the collection of parameters for a generic ASCD model. When needed, we will explicitly mention the innovation distribution to which $\theta$ corresponds. By Bayes' Theorem, the joint conditional distribution of $\theta$ and $\mathbf{h}$ is given by:

$$
f(\theta, \mathbf{h} \mid \mathbf{y}) \propto f(\mathbf{y} \mid \mathbf{h}, \theta) f(\mathbf{h} \mid \theta) f(\theta),
$$

where $f(\mathbf{y} \mid \mathbf{h}, \theta)$ is the likelihood of $\mathbf{y}$ given $(\theta, \mathbf{h}), f(\mathbf{h} \mid \theta)$ is the density of $\mathbf{h}$ and $f(\theta)$ is the prior density of $\theta$. Bayesian inference of $(\theta, \mathbf{h})$ is based upon the posterior distribution $f(\theta, \mathbf{h} \mid \mathbf{y})$.

The ASCD model is completed by specifying proper prior distributions for the parameters of the model. For simplicity, we assume that all prior distributions of the parameters in the model are mutually independent. The prior distributions of $\mu$ and $\psi$ are chosen to be $\mu \sim \mathcal{N}(0,10)$ and $\psi \sim \mathcal{N}(0,10)$, respectively. To impose weak stationary condition on the latent process, the prior distribution of $\phi$ follows a normal distribution $\phi \sim \mathcal{N}(0,10)$ truncated in the interval $(-1,1)$. These prior distributions result in reasonably flat densities over their support regions. In the MCMC algorithm, we sample $\sigma^{2}$ instead of $\sigma$, and for this we use an inverse Gamma distribution $\mathcal{I} \mathcal{G}(5,0.05)$. The same prior is used in Pitt and Shephard (1999a). It is seen that except for $\phi$ all the above prior distributions are conjugate, which is convenient for the calculation of the posterior distributions or the full conditionals ${ }^{1}$. For the prior distribution of $\gamma$ and $v$, we use either the Cauchy prior with the density

$$
p(\gamma)=\frac{1}{1+\gamma^{2}}
$$

\footnotetext{
${ }^{1}$ The full conditional of a parameter is defined as the conditional distribution given that other parameters in the model have been previously sampled.
} 
which is also used in Bauwens and Laubrano (1998), or the exponential distribution used in Geweke (1993). In our implementation of the method, we obtained very similar results for the two priors.

An outline of the MCMC algorithm is listed in Table 1. Below we provide additional explanations.

Table 1: MCMC algorithm for the ASCD model.

Step 0. Initialize h, $\mu, \phi, \psi, \sigma$, and $v$ or $\gamma$.

Step 1. Sample $h_{t}, t=1, \ldots, T$.

Step 2. Sample $\phi$.

Step 3. Sample $\mu, \psi$, and $\sigma^{2}$.

Step 4. Sample $v$ or $\gamma$.

Step 5. Go to Step 1.

Step 0. Initialize $\mathbf{h}, \mu, \phi, \psi, \sigma, v$ and $\gamma$. To start the MCMC algorithm, the parameters of the model are set as $\mu=-0.5, \phi=0.5, \psi=0.5, \sigma=0.12$, $v=0.5$ and $\gamma=0.5$. The initial values of $\mathbf{h}$ are generated from the latent $\mathrm{AR}(1)$ process with the above initial parameters.

Step 1. Sample h. The simulation is conducted via a single-move $\mathrm{MH}$ method. The full conditionals of the latent random variables can be expressed as follows:

$$
\begin{aligned}
f\left(h_{1} \mid \mathbf{y}, h_{2}, \theta\right) & \propto f\left(y_{1} \mid h_{1}\right) f\left(h_{1} \mid \theta\right) f\left(h_{1} \mid h_{2}, y_{1}, \theta\right), \\
f\left(h_{t} \mid \mathbf{y}, h_{t-1}, h_{t+1}, \theta\right) & \propto f\left(y_{t} \mid h_{t}\right) f\left(h_{t} \mid h_{t-1}, y_{t-1}, \theta\right) f\left(h_{t} \mid h_{t+1}, y_{t}, \theta\right), \\
f\left(h_{T} \mid \mathbf{y}, h_{T-1}, \theta\right) & \propto f\left(y_{T} \mid h_{T}\right) f\left(h_{T} \mid h_{T-1}, y_{T-1}, \theta\right),
\end{aligned}
$$

where $f\left(y_{t} \mid h_{t}\right), t=1, \ldots, T$, are the conditional densities of the durations and $f\left(h_{1} \mid \theta\right)$ is the density of the latent log duration $h_{1} \cdot f\left(h_{t} \mid h_{t-1}, y_{t-1}, \theta\right)$ and $f\left(h_{t} \mid h_{t+1}, y_{t}, \theta\right)$ are the conditional densities of $h_{t}$ given $h_{t-1}$ and of $h_{t}$ given $h_{t+1}$, respectively. They can be obtained by using the latent equation (14). Since $y_{T}$ is the last observation, the posterior distribution of $h_{T}$ depends only on $y_{T}, y_{T-1}$, and $h_{T-1}$. 
Below we describe the simulation algorithm for the full conditionals of $h_{t}, t=2, \ldots, T-1$. Since the simulations of $h_{t}$ for different distributions of the innovations are similar, we present the algorithm only for the Weibull( $v$, 1) distribution with or without the leverage effect.

\section{Sampling $h_{t}$ under the Weibull $(v, 1)$ distribution without "leverage effect".}

The full conditional of $h_{t}$ can be represented as follows:

$$
\begin{aligned}
& f\left(h_{t} \mid \mathbf{y}, h_{t-1}, h_{t+1}, \theta_{W}\right) \\
& \propto f\left(y_{t} \mid h_{t}\right) f\left(h_{t} \mid h_{t-1}, y_{t-1}, \theta_{W}\right) f\left(h_{t} \mid h_{t+1}, y_{t}, \theta_{W}\right) \\
& \propto \exp \left(-v h_{t}\right) y_{t}^{v-1} \exp \left\{-y_{t}^{v} \exp \left(-v h_{t}\right)\right\} \\
& \quad \times \exp \left\{-\frac{\left[\left(h_{t}-\mu\right)-\phi\left(h_{t-1}-\mu\right)\right]^{2}}{2 \sigma^{2}}\right\} \\
& \quad \times \exp \left\{-\frac{\left[\left(h_{t+1}-\mu\right)-\phi\left(h_{t}-\mu\right)\right]^{2}}{2 \sigma^{2}}\right\} \\
& \propto \exp \left(-v h_{t}\right) \exp \left\{-y_{t}^{v} \exp \left(-v h_{t}\right)\right\} \\
& \quad \times \exp \left\{-\frac{\left(h_{t}-\mu_{t}\right)^{2}}{2 b}\right\},
\end{aligned}
$$

where

$$
\begin{aligned}
a_{t} & =\mu+\frac{\phi\left[\left(h_{t}-\mu\right)+\left(h_{t+1}-\mu\right)\right]}{1+\phi^{2}}, \\
b & =\frac{\sigma^{2}}{1+\phi^{2}} .
\end{aligned}
$$

For convenience, the full conditionals of $h_{1}$ and $h_{T}$ are given in Appendix B. It it noticed that in (18) the posterior distribution is not a simple distribution but proportional to a product of three positive functions. Therefore, this full conditional can not be simulated directly. For this reason, to generate variates from the full conditional we employ the slice sampler method introduced in Edwards and Sokal (1998) and Neal (2003). It is easy to verify that the product in (18) can be expressed as:

$$
g\left(h_{t}\right) \propto \exp \left\{-y_{t}^{v} \exp \left(-v h_{t}\right)\right\} \exp \left\{-\frac{\left(h_{t}-\mu_{t}\right)^{2}}{2 b}\right\},
$$


where $\mu_{t}=a_{t}-b v$. Therefore, we have the following algorithm for the slice sampler for $h_{t}$ :

SS0. Initialize $h_{t}^{(0)}$, Set $n=0$.

SS1. Draw $u_{1}$ uniformly from the interval $\left(0, \exp \left\{-y_{t}^{v} \exp \left(-v h_{t}^{(n)}\right)\right\}\right)$. Define an interval for $h_{t}$ through the inequality

$$
u_{1} \leq \exp \left\{-y_{t}^{v} \exp \left(-v h_{t}\right)\right\}
$$

which is equivalent to

$$
h_{t} \geq-\log \left(\frac{y_{t}}{\left(-\log \left(u_{1}\right)\right)^{\frac{1}{v}}}\right) .
$$

SS2. Draw $u_{2}$ uniformly from the interval $\left(0, \exp \left\{-\frac{\left(h_{t}^{(n)}-\mu_{t}^{(n)}\right)^{2}}{2 b}\right\}\right)$, where $\mu_{t}^{(n)}$ is calculated from (21). Define another interval for $h_{t}$ through the inequality

$$
u_{2}<\exp \left\{-\frac{\left(h_{t}-\mu_{t}^{(n)}\right)^{2}}{2 b}\right\}
$$

which is equivalent to

$$
\mu_{t}^{(n)}-\sqrt{-2 b \log \left(u_{2}\right)} \leq h_{t} \leq \mu_{t}^{(n)}+\sqrt{-2 b \log \left(u_{2}\right)} .
$$

SS3. Draw $h_{t}^{(n+1)}$ uniformly from the interval determined by (22) and (23):

$$
\begin{aligned}
& h_{t}^{(n+1)} \\
& \sim \mathcal{U}\left(\max \left\{-\log \left(\frac{y_{t}}{\left(-\log \left(u_{1}\right)\right)^{\frac{1}{v}}}\right), \mu_{t}^{(n)}-\sqrt{-2 b \log \left(u_{2}\right)}\right\}, \mu_{t}^{(n)}+\sqrt{-2 b \log \left(u_{2}\right)}\right)
\end{aligned}
$$

SS4. Stop, if a stopping criterion is met; otherwise, set $n+1$ and repeat from SS1. 
To start the slice sampling procedure, the sampled value of $h_{t}$ from the last MCMC step is set as the initial value. Since the full conditionals of $h_{t}$ in each MCMC step are similar, this initial value is considered to be a good starting point. As the slice sampler adapts to the form of the density function of the underlying variable, it is deemed to be more efficient than other samplers. Under certain technical conditions, Roberts and Rosenthal (1999) show that the slice algorithm is robust and has geometric periodicity properties. Mira and Tierney (2002) prove that the slice sampler has a smaller second-largest eigenvalue, which ensures faster convergence to the underlying distribution. This is a critical property for our implementation, where typically it suffices to use just five iterations of the algorithm.

\section{Sampling $h_{t}$ under the Weibull $(v, 1)$ distribution with "leverage effect".}

In this case, the full conditional of $h_{t}$ is

$$
\begin{aligned}
& f\left(h_{t} \mid \mathbf{y}, h_{t-1}, h_{t+1}, \theta_{W}\right) \\
& =c_{1 t} f\left(y_{t} \mid h_{t}\right) f\left(h_{t} \mid h_{t-1}, y_{t-1}, \theta_{W}\right) f\left(h_{t} \mid h_{t+1}, y_{t}, \theta_{W}\right) \\
& =c_{2 t} \exp \left(-v h_{t}\right) y_{t}^{v-1} \exp \left\{-y_{t}^{v} \exp \left(-v h_{t}\right)\right\} \\
& \quad \times \exp \left\{-\frac{\left[\left(h_{t}-\mu\right)-\phi\left(h_{t-1}-\mu-\psi y_{t-1} \exp \left(-h_{t-1}\right)\right]^{2}\right.}{2 \sigma^{2}}\right\} \\
& \quad \times \exp \left\{-\frac{\left[\left(h_{t+1}-\mu\right)-\phi\left(h_{t}-\mu-\mu-\psi y_{t} \exp \left(-h_{t}\right)\right]^{2}\right.}{2 \sigma^{2}}\right\} \\
& =c_{2 t} \exp \left\{-\frac{\left[\left(h_{t+1}-\mu\right)-\phi\left(h_{t}-\mu\right)-\psi \exp \left(-h_{t} / 2\right) y_{t}\right]^{2}}{2 \sigma^{2}}\right\} \times g\left(h_{t}\right),
\end{aligned}
$$

where $c_{1 t}$ and $c_{2 t}$ are the two normalizing constants, and

$$
\begin{aligned}
g\left(h_{t}\right)= & \exp \left(-v h_{t}\right) \exp \left\{-y_{t}^{v} \exp \left(-v h_{t}\right)\right\} \\
& \times \exp \left\{-\frac{\left[\left(h_{t}-\mu\right)-\phi\left(h_{t-1}-\mu\right)-\psi \exp \left(-h_{t-1} / 2\right) y_{t-1}\right]^{2}}{2 \sigma^{2}}\right\} .
\end{aligned}
$$

It is easy to see that the full conditional of $h_{t}$ is bounded above by $c_{2 t} g\left(h_{t}\right)$. Like in the case without the leverage effect, also here we can use the MH 
algorithm to simulate the full conditional with the proposal proportional to $g\left(h_{t}\right)$, which can be sampled using the slice sampler. The advantage of the slice sampler is that even a few iterations can give us a good result. This is not the case with the crude $\mathrm{MH}$ algorithm, where many generated points have to be discarded.

Step 2. Sample $\phi$. Given a truncated univariate normal prior distribution $\phi \sim \mathcal{N}\left(\alpha_{\phi}, \beta_{\phi}^{2}\right)$, the full conditional of $\phi$ is

$$
\begin{aligned}
f\left(\phi \mid \mathbf{y}, \mu, \psi, \sigma^{2}\right) & \propto p\left(h_{1} \mid \theta\right) \prod_{t=1}^{T-1} p\left(h_{t+1} \mid h_{t}, \theta, y_{t}\right) \exp \left\{-\frac{\left(\phi-\alpha_{\phi}\right)^{2}}{2 \beta_{\phi}^{2}}\right\} \\
& \propto \mathcal{N}\left(\frac{d}{c}, \frac{1}{c}\right)\left(1-\phi^{2}\right)^{\frac{1}{2}}
\end{aligned}
$$

where

$$
\begin{aligned}
& c=\frac{-\left(h_{1}-\mu\right)^{2}+\sum_{t=1}^{T-1}\left(h_{t}-\mu\right)^{2}}{\sigma^{2}}+\frac{1}{\beta_{\phi}^{2}}, \\
& d=\frac{\sum_{t=1}^{T-1}\left(h_{t}-\mu\right)\left(h_{t+1}-\mu\right)}{\sigma^{2}}+\frac{\alpha_{\phi}}{\beta_{\phi}^{2}} .
\end{aligned}
$$

For convenience, the full conditional is detailed in Appendix B. It is proportional to the product of a univariate normal distribution and a positive function, and therefore it can be sampled by the slice sampler.

Step 3. Sample parameters $\mu, \psi$ and $\sigma^{2}$. Since the prior distributions for $\mu$ and $\psi$ are conjugate normals and for $\sigma^{2}$ an inverse Gamma distribution, the sampling can be done relatively easily. The details of these full conditionals are presented in Appendix B.

Step 4. Sample parameter $v$ or $\gamma$. Here we only give the sampling procedure for the parameter $v$. The sampling of $\gamma$ can be performed in the same fashion. The full conditional of $v$ is given by:

$$
\begin{aligned}
f\left(v \mid \mathbf{y}, \mathbf{h}, \mu, \phi, \sigma^{2}\right) & \propto f(\mathbf{y} \mid \mathbf{h}, v) f(v) \\
& =f(v) \prod_{t=1}^{T} v y_{t}^{v-1} \exp \left(-v h_{t}\right) \exp \left(-\frac{y_{t}^{v}}{\exp \left(v h_{t}\right)}\right),
\end{aligned}
$$

where $f(v)$ is a prior density of $v$. In the literature, several specifications of this prior distribution have been proposed. Similarly to Bauwens and 
Laubrano (1998), in our implementation we adapt the Cauchy prior. Since it is not possible to sample the full conditional directly, we use a random-walk $\mathrm{MH}$ algorithm, where the proposal density is the standard Gaussian density and the acceptance probability is computed using the equation (26).

\subsection{Particle filter}

To perform a model comparison, we need to evaluate the model likelihood. For the ASCD models, the likelihoods are difficult to compute analytically due to their non-linear structure. Therefore, to perform this task we employ an auxiliary particle filter proposed by Pitt and Shephard (1999b). This is an efficient recursive algorithm that approximates the filter and the one-stepahead prediction distributions. By successive conditioning, we can represent the sample likelihood of the ASCD model as follows:

$$
f(\mathbf{y} \mid \theta)=f\left(y_{1} \mid \theta\right) \prod_{t=2}^{T} f\left(y_{t} \mid \mathcal{F}_{t-1}, \theta\right),
$$

where $\mathcal{F}_{t}=\sigma\left(y_{1}, \ldots, y_{t}\right)$ is the information known at time $t$. The conditional density of $y_{t+1}$ given $\theta$ and $\mathcal{F}_{t}$ has the following representation:

$$
\begin{aligned}
f\left(y_{t+1} \mid \mathcal{F}_{t}, \theta\right) & =\int f\left(y_{t+1} \mid h_{t+1}, \theta\right) d F\left(h_{t+1} \mid \mathcal{F}_{t}, \theta\right) \\
& =\int f\left(y_{t+1} \mid h_{t+1}, \theta\right) f\left(h_{t+1} \mid h_{t}, \theta\right) d F\left(h_{t} \mid \mathcal{F}_{t}, \theta\right) .
\end{aligned}
$$

Suppose that we have a particle sample $\left\{h_{t}^{(i)}, i=1, \ldots, N\right\}$ of $h_{t}$ from the filtered distribution of $\left(h_{t} \mid \mathcal{F}_{t}, \theta\right)$ with weights $\left\{\pi_{i}, i=1, \ldots, N\right\}$ such that $\sum_{i=1}^{N} \pi_{i}=1$. Given this sample, the one-step-ahead predictive density of $h_{t+1}$ is given by:

$$
f\left(h_{t+1} \mid \mathcal{F}_{t}, \theta\right) \approx \sum_{i=1}^{N} \pi_{i} f\left(h_{t+1} \mid h_{t}^{(i)}, \theta\right) .
$$

Then the one-step-ahead predictive distribution of $h_{t+1}$ can be sampled and the conditional density (28) can be evaluated numerically by:

$$
f\left(y_{t+1} \mid \mathcal{F}_{t}, \theta\right) \approx \sum_{i=1}^{N} \pi_{i} f\left(y_{t+1} \mid h_{t+1}^{(i)}, \theta\right)
$$


where $h_{t+1}^{(i)}$ are particles from the predictive distribution of $\left(h_{t+1} \mid \mathcal{F}_{t}, \theta\right)$. For the approximation (29) to be feasible, the predictive density of $h_{t+1}$ must be known. This assumption is satisfied in our context, since the form of the latent $\mathrm{AR}(1)$ process implies that $h_{t+1}$ has a conditional normal distribution $h_{t+1} \sim \mathcal{N}\left(\mu+\phi\left(h_{t}-\mu\right)+\psi y_{t} \exp \left(-h_{t}\right), \sigma^{2}\right)$. This fact is also useful for forecasting.

Now the question is how to sample $\left(h_{t+1} \mid \mathcal{F}_{t+1}, \theta\right)$ given that we have a particle sample from the filter distribution of $\left(h_{t} \mid \mathcal{F}_{t}, \theta\right)$. Below we present an algorithm for the ASCD models based on the procedure suggested by Chib, Nardari, and Shephard (2006):

PF1. Given a sample $\left\{h_{t}^{(i)}, i=1, \ldots, N\right\}$ from $\left(h_{t} \mid \mathcal{F}_{t}, \theta\right)$, calculate the expectation $\hat{h}_{t+1}^{*(i)}=\mathrm{E}\left(h_{t+1} \mid h_{t}^{(i)}\right)$ and

$$
\pi_{i}=f\left(y_{t+1} \mid \hat{h}_{t+1}^{*(i)}, \theta\right), i=1, \ldots, N .
$$

Sample $N$ times with replacement the integers $1, \ldots, N$ with probabilities given by $\hat{\pi}_{i}=\pi_{i} / \sum_{i=1}^{N} \pi_{i}$. Denote the sampled indexes by $n_{1}, \ldots, n_{N}$ and associate these with particles $\left\{h_{t}^{\left(n_{1}\right)}, \ldots, h_{t}^{\left(n_{N}\right)}\right\}$.

PF2. For each value $n_{i}$ from Step PF1, sample the values $\left\{h_{t+1}^{*(1)}, \ldots, h_{t+1}^{*(N)}\right\}$ using

$$
h_{t+1}^{*(i)}=\mu+\phi\left(h_{t}^{\left(n_{i}\right)}-\mu\right)+\psi \exp \left(-h_{t}^{\left(n_{i}\right)} / 2\right) y_{t}+\sigma v_{t+1}, i=1, \ldots, N .
$$

where $v_{t+1} \sim \mathcal{N}(0,1)$.

PF3. Calculate the weights of the values $\left\{h_{t+1}^{*(1)}, \ldots, h_{t+1}^{*(N)}\right\}$ as

$$
\pi_{i}^{*}=\frac{f\left(y_{t+1} \mid h_{t+1}^{*(i)}, \theta\right)}{f\left(y_{t+1} \mid \hat{h}_{t+1}^{*(i)}, \theta\right)}, \quad i=1, \ldots, N,
$$

and using these weights resample the values $\left\{h_{t+1}^{*(1)}, \ldots, h_{t+1}^{*(N)}\right\} N$ times with replacement to obtain a sample $\left\{h_{t+1}^{(1)}, \ldots, h_{t+1}^{(N)}\right\}$ from the filter distribution of $\left(h_{t+1} \mid \mathcal{F}_{t+1}, \theta\right)$. In our implementation we used $N=$ 3000 . 


\subsection{Diagnostics}

There are a number of tools that can be used to assess the goodness-of-fit of our ASCD models. One of them is the Kolmogorov-Smirnov (KS) test, which is designed to examine whether realized observation errors originated from the assumed distribution. Another approach is to analyze the Probability Integral Transforms (PITs), which was proposed in Diebold, Guther, and Tay (1998).

Suppose that $\left\{f\left(y_{t} \mid \mathcal{F}_{t-1}\right)\right\}_{t=1}^{T}$ is a sequence of conditional densities of $y_{t}$ and $\left\{p\left(y_{t} \mid \mathcal{F}_{t-1}\right)\right\}_{t=1}^{T}$ is the corresponding sequence of one-step-ahead density forecasts. The PIT of $y_{t}$ is defined as

$$
u(t)=\int_{-\infty}^{y_{t}} p\left(z \mid \mathcal{F}_{t-1}\right) d z .
$$

Under a null hypothesis that the sequence $\left\{p\left(y_{t} \mid \mathcal{F}_{t-1}\right)\right\}_{t=1}^{T}$ coincides with $\left\{f\left(y_{t} \mid \mathcal{F}_{t-1}\right)\right\}_{t=1}^{T}$, the sequence $\{u(t)\}_{t=1}^{T}$ corresponds to i.i.d. observations from the uniform distribution on $[0,1]$. In our univariate ASCD models, the PITs can be calculated using the following formulas.

The ASCD-G model:

$$
\begin{aligned}
u(t) & \approx \frac{1}{N} \sum_{i=1}^{N} \int_{-\infty}^{y_{t}} \frac{1}{\Gamma(\gamma)} z^{\gamma-1} \exp \left(-\gamma h_{t}^{(i)}\right) \exp \left\{-\frac{z}{\exp \left(h_{t}^{(i)}\right)}\right\} d z \\
& =\frac{1}{N} \sum_{i=1}^{N} \frac{1}{\Gamma(\gamma)} g\left(\gamma, y_{t} \exp \left(-h_{t}^{(i)}\right)\right)
\end{aligned}
$$

where $g\left(\gamma, y_{t} \exp \left(-h_{t}\right)\right)$ is the incomplete gamma function.

The ASCD-W model:

$$
\begin{aligned}
u(t) & \approx \frac{1}{N} \sum_{i=1}^{N} \int_{-\infty}^{y_{t}} v z^{v-1} \exp \left(-v h_{t}^{(i)}\right) \exp \left\{-\left(\frac{z}{\exp \left(h_{t}^{(i)}\right)}\right)^{v}\right\} d z \\
& =1-\frac{1}{N} \sum_{i=1}^{N} \exp \left\{-\left(\frac{y_{t}}{\exp \left(-h_{t}^{(i)}\right)}\right)^{v}\right\}
\end{aligned}
$$


The ASCD-E model

$$
\begin{aligned}
u(t) & \approx \frac{1}{N} \sum_{i=1}^{N} \int_{-\infty}^{y_{t}} \exp \left(-h_{t}^{(i)}\right) \exp \left\{-\frac{z}{\exp \left(h_{t}^{(i)}\right)}\right\} d z \\
& =1-\frac{1}{N} \sum_{i=1}^{N} \exp \left\{-\frac{y_{t}}{\exp \left(-h_{t}^{(i)}\right)}\right\} .
\end{aligned}
$$

In the computation of $u(t), h_{t}^{(i)}$ are particles from the corresponding predictive distribution of $h_{t}$ with weights $1 / N$.

\subsection{Model selection}

In the literature, there are several ways to conduct a model selection. In general, the AIC in Akaike (1987) and BIC in Schwarz (1978) criteria are commonly used for SV and GARCH models. It is well known that both AIC and BIC need the exact number of parameters in the model. This is not the case for the SCD models. In the Bayesian inference of the SCD model defined in this paper, the latent states are augmented as parameters. We can not treat each state as an independent parameter since all the states are highly correlated. For instance, if we fit a data set with $T$ observations using the ASCD model with Gamma innovations, the total number of parameters could be up to $(T+5)$, where 5 counts the number of parameters indexing the model. Thus, there is ambiguity in using AIC or BIC for model selection in the context of the SCD models. Moreover, by definition, AIC always prefers models with larger number of parameters. In contrast, BIC will select a model with small number of parameters. Consequently, the number of parameters could affect the final choice of the model. Based on this concerns, a new criterion for model comparison, called deviance information criterion (DIC), has been introduced by Spiegelhalter, Best, Carlin, and Van der Linde (2002). DIC is particularly useful for hierarchical models such as the SV and SCD models. Berg, Meyer, and Yu (2004) use this criterion for model comparison of univariate SV models. The DIC criterion does not depend on the number of model parameters directly and is defined by the following formula:

$$
\mathrm{DIC}=\bar{D}+P_{D} .
$$

The first term $\bar{D}$ is a Bayesian measure of model fit, which is defined as the posterior mean of the deviance

$$
\bar{D}(\theta)=E_{\theta \mid \mathbf{y}}[D(\theta)],
$$


where $D(\theta)=-2 \log f(\mathbf{y} \mid \theta)$, and $\theta$ and $\mathbf{y}$ represent the model parameters and the data, respectively. Larger values of $\bar{D}$ indicate deterioration of the model fit. The second term is defined as

$$
\begin{aligned}
P_{D} & =\bar{D}-D(\bar{\theta}) \\
& =E_{\theta \mid \mathbf{y}}[D(\theta)]-D\left(E_{\theta} \mid \mathbf{y}[\theta]\right),
\end{aligned}
$$

where $D(\bar{\theta})$ is the deviance of the posterior mean, which measures the complexity of the model. In other words, $P_{D}$ is a difference between the posterior mean of the deviance of the model and the deviance under the posterior mean of $\theta$. The larger the $P_{D}$ is, the easier it is for the model to fit the data. The term $P_{D}$ is usually called the effective number of parameters.

Since the likelihood is usually not available for the ASCD models, to compute DIC we have to use numerical methods to evaluate $\bar{D}$ and $D(\bar{\theta})$. In this paper, we use the MCMC output to calculate $\bar{D}$, and the APF for the calculation of $D(\bar{\theta})$. As the true value of $\theta$ is not known in advance, the Bayesian estimate $\bar{\theta}$ of $\theta$ has to be used instead. For a given model, we use the following formula to compute $\bar{D}(\theta)$

$$
\begin{aligned}
\bar{D}(\theta) & =E_{\theta \mid \mathbf{y}}[-2 \log f(\mathbf{y} \mid \theta)] \\
& =E_{\theta \mid \mathbf{y}}\left[\log f\left(y_{1} \mid h_{1}, \theta\right)+\sum_{t=2}^{T} f\left(y_{t} \mid h_{t}, \theta\right)\right] \\
& =\frac{1}{N-n} \sum_{i=n}^{N}\left[\log f\left(y_{1} \mid h_{1}^{(i)}, \theta^{(i)}\right)+\sum_{t=2}^{T} f\left(y_{t} \mid h_{t}^{(i)}, \theta^{(i)}\right)\right],
\end{aligned}
$$

where $h_{t}^{(i)}$ and $\theta^{(i)}$ are the draws from their full conditionals during the MCMC process, $n$ is the length of the burn-in period in the MCMC estimation process, and $N$ is the number of iterations. Regarding the $D(\bar{\theta})$ term, we use the APF method for the calculation, where $\bar{\theta}$ is the estimate of $\theta$ from the MCMC estimation procedure. The objective of the particle filter here is to obtain samples of $h_{t}$ conditional on the $h_{t-1}$ given $\bar{\theta}$.

\section{Simulation studies}

In this section, we present results of our simulation studies for the ASCD model with and without the leverage effect. After each model is fitted, we use the KS test to check whether the fitted model agrees with the generated 
duration time series. For given $\theta$, the following equations are used to generate the duration time series $\mathbf{y}$ and the states $\mathbf{h}$ :

$$
\begin{aligned}
h_{t+1} & \sim \mathcal{N}\left(\mu+\phi\left(h_{t}-\mu\right)+\psi y_{t} \exp \left(-h_{t}\right), \sigma^{2}\right), \\
y_{t} & \sim \exp \left(h_{t}\right) \epsilon_{t},
\end{aligned}
$$

where $h_{1} \sim \mathcal{N}\left(\mu, \sigma^{2} /\left(1-\phi^{2}\right)\right), y_{T} \sim \exp \left(h_{T}\right) \epsilon_{T}$ and $\epsilon_{t}$ follows one of the three innovation distributions.

The true parameters used to generate the durations are presented in the second column of Tables 2 and 3 for the various specified ASCD models. We generated 5,000 observations from each of the ASCD model. The first 4,000 observations were fitted by the ASCD model and the last 1,000 observations were used for comparison with the one-step-ahead out-of-sample duration forecast. Our proposed estimation algorithm was iterated 100,000 iterations and the first 20,000 sampled points were discarded as the burn-in before Bayesian inference was drawn. In Tables 2 and 3 we present the estimated parameters together with the Bayesian highest probability density (HPD) confidence intervals and standard errors. It can be seen that the estimates and the true values of the parameters are very close.

Table 2: True and estimated parameters of the uncorrelated SCD model with different

\begin{tabular}{|c|c|c|c|}
\hline Parameter & True & Est. & \\
\hline
\end{tabular}
innovation distributions based on the generated duration data.

$\begin{array}{ccccc}\text { Panel A: } \operatorname{Gamma}(\gamma, \mathbf{1}) & & & & \\ \phi & \mathbf{0 . 9 4} & 0.9328 & 0.0120 & (0.9094,0.9556) \\ \sigma & \mathbf{0 . 1 9} & 0.1921 & 0.0189 & (0.1548,0.2280) \\ \gamma & \mathbf{0 . 9 4} & 0.9515 & 0.0191 & (0.9155,0.9903)\end{array}$

Panel B: Weibull $(v, 1)$

$\begin{array}{lllll}\phi & \mathbf{0 . 9 4} & 0.9569 & 0.0078 & (0.9417,0.9719) \\ \sigma & \mathbf{0 . 1 9} & 0.1794 & 0.0161 & (0.1488,0.2125) \\ v & \mathbf{0 . 9 4} & 0.9377 & 0.0133 & (0.9125,0.9644)\end{array}$

Panel C: $\operatorname{Exp}(1)$

\begin{tabular}{lllll}
$\phi$ & $\mathbf{0 . 9 4}$ & 0.9456 & 0.0084 & $(0.9288,0.9613)$ \\
$\sigma$ & $\mathbf{0 . 1 9}$ & 0.2004 & 0.0150 & $(0.1716,0.2302)$ \\
\hline
\end{tabular}


Table 3: True and estimated parameters of the ASCD model based on the generated duration data.

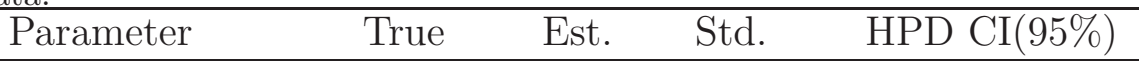

Panel A: $\operatorname{Gamma}(\gamma, 1)$

$\begin{array}{lrrrr}\phi & \mathbf{0 . 9 4} & 0.9387 & 0.0095 & (0.9198,0.9566) \\ \psi & \mathbf{- 0 . 4 1} & -0.4200 & 0.0328 & (-0.4845,-0.3545) \\ \sigma & \mathbf{0 . 1 9} & 0.1769 & 0.0203 & (0.1405,0.2153) \\ \gamma & \mathbf{0 . 9 4} & 0.9304 & 0.0196 & (0.8925,0.9681)\end{array}$

Panel B: Weibull $(v, 1)$

$\begin{array}{lrrrr}\phi & \mathbf{0 . 9 4} & 0.9417 & 0.0114 & (0.9168,0.9642) \\ \psi & \mathbf{- 0 . 4 1} & -0.4392 & 0.0460 & (-0.5209,-0.3526) \\ \sigma & \mathbf{0 . 1 9} & 0.1826 & 0.0246 & (0.1332,0.2372) \\ v & \mathbf{0 . 9 4} & 0.9471 & 0.0171 & (0.9133,0.9800)\end{array}$

Panel C: $\operatorname{Exp}(1)$

\begin{tabular}{lrrrr}
$\phi$ & $\mathbf{0 . 9 4}$ & 0.9247 & 0.0089 & $(0.9070,0.9422)$ \\
$\psi$ & $\mathbf{- 0 . 4 1}$ & -0.4384 & 0.0317 & $(-0.5000,-0.3769)$ \\
$\sigma$ & $\mathbf{0 . 1 9}$ & 0.2211 & 0.0174 & $(0.1870,0.2544)$ \\
\hline
\end{tabular}


The overall model fit is assessed through the analysis of the PITs obtained from the formulas (35)-(37). All of the six specified SCD modes passed the $\mathrm{KS}$ test. Here we only present the test results for the ASCD-G model. The uniform distribution of $u(t)$ on the interval $[0,1]$ is visualized in Figure 1 by the scatter plot and histogram. The KS test statistic is 0.0214 with the corresponding $p$-value of 0.444 . Based on these values, we do not reject the null hypothesis that the PITs are uniformly distributed over the interval $[0,1]$ at the $5 \%$ significance level. In Figure 2 the empirical cumulative distribution function $(\mathrm{CDF})$ of the PITs is depicted together with the theoretical CDF of the uniform $[0,1]$. The graph confirms our earlier assessment that the fitted ASCD-G model agrees well with the generated data. From the above comparisons, and the result of the KS test, we conclude that the proposed slice sampler within the MCMC approach for the ASCD-G model fits well the simulated transaction data.
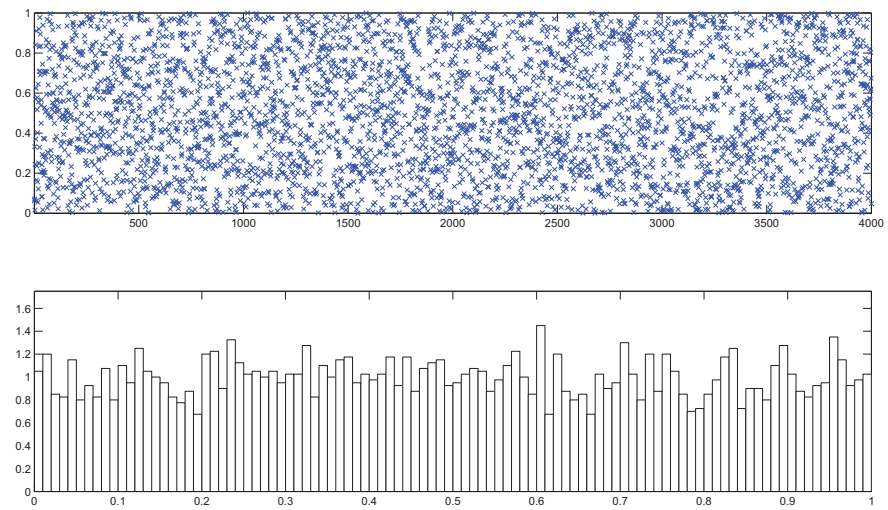

Figure 1: Analysis of the PITs from the ASCD-G model based on the simulated data. The top panel shows the scatter plot of $u(t)$ while the bottom the histogram of $u(t)$.

Once the ASCD models were estimated, we used them to perform insample and out-of-sample one-step-ahead forecasts. In Figures 3 to 5 we compare the generated durations with the estimated and one-step-ahead insample and out-sample forecasted durations, where the latter were separated by a vertical dotted line. The forecasted durations appear to resemble closely the true time series of the generated durations. 


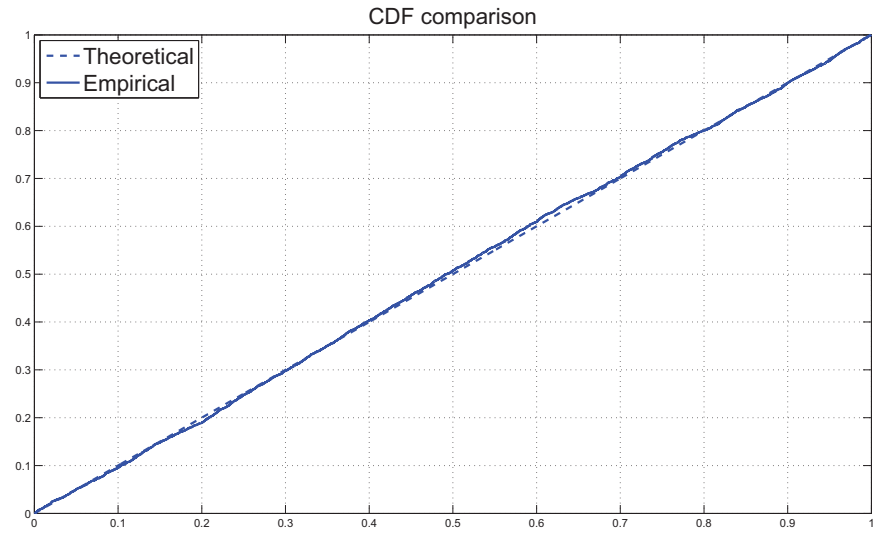

Figure 2: Comparison between the theoretical uniform CDF and the empirical CDF of the PITs from the ASCD-G model based on the generated duration data.
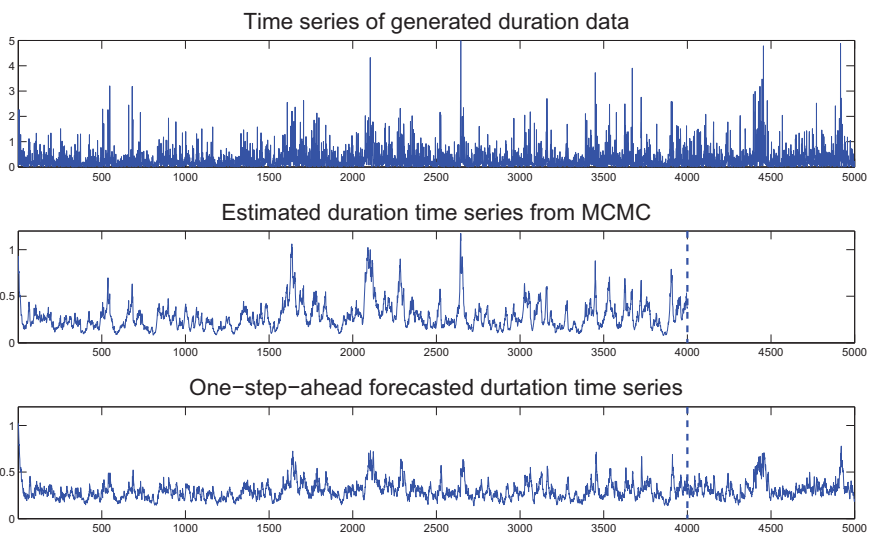

Figure 3: Comparison between true and one-step-ahead forecasted duration time series under the ASCD-W model based on the generated transaction data. 

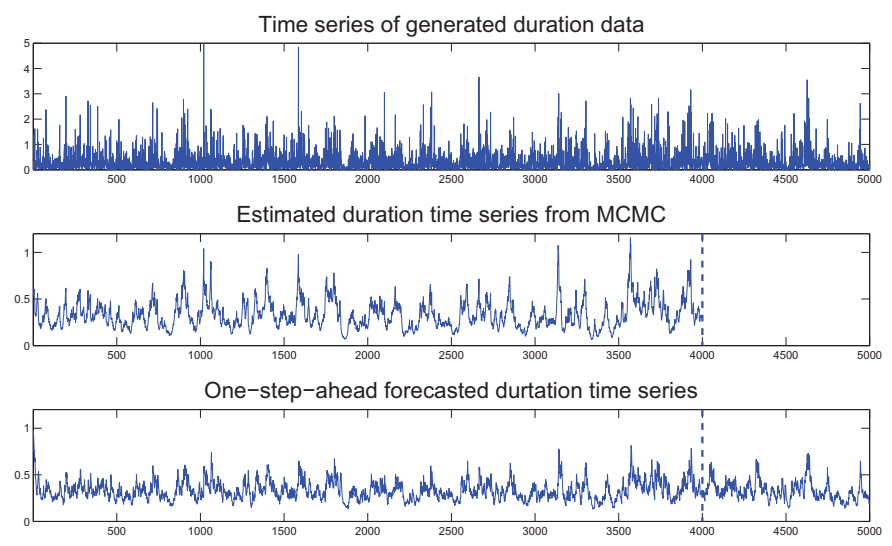

Figure 4: Comparison between true and one-step-ahead forecasted duration time series under the ASCD-G model based on the generated transaction data.
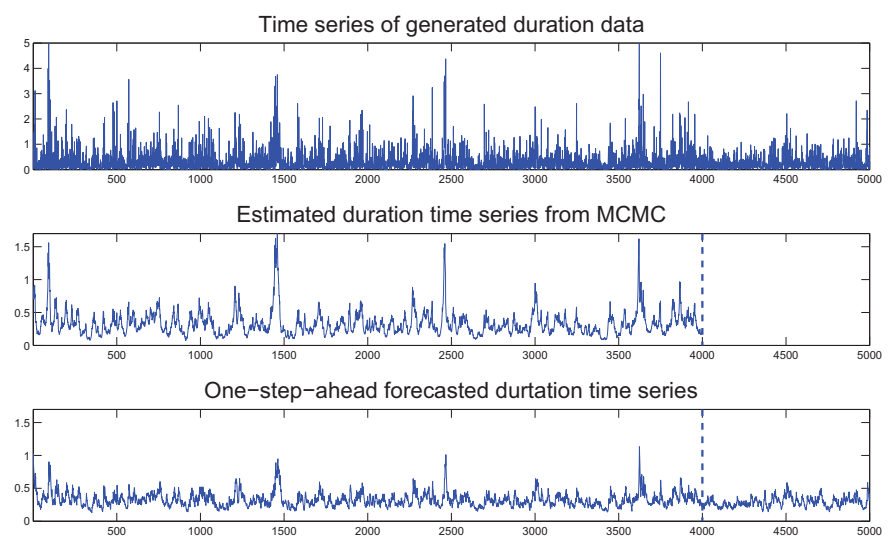

Figure 5: Comparison between true and one-step-ahead forecasted duration time series under the ASCD-E model based on the generated transaction data. 


\section{Empirical Analysis}

In this section, we apply the proposed ASCD models to a duration data set of the IBM transactions. It covers the period from November 1, 1990, to January 31, 1990, yielding 24,765 transactions. This data set has been analyzed, among others, by Knight and Ning (2008), Feng, Jiang, and Song (2004) and Xu, Knight, and Wirjanto (2011). The reason for using this data set is to facilitate comparison of our estimation results with those from prior studies.

Tables 4 and 5 report the estimated parameters for the SCD model with and without the leverage effect based on the three types of observation innovations. Estimates of the common parameters of the different SCD models are very close to each other, with small estimation errors due presumably to the large sample size. One noticable finding is that the estimated correlation coefficient between the observation and latent $\mathrm{AR}(1)$ processes is negative. We interpret this to mean that the IBM duration time series has a leverage effect with its log duration dynamic. This finding is in contrast to the results reported in Feng, Jiang, and Song (2004) and Xu, Knight, and Wirjanto (2011), where a positive correlation coefficient is found between the log transformed duration time series and the latent time series.

Table 4: True and estimated parameters of the uncorrelated SCD model based on the IBM duration data.

\begin{tabular}{crrr}
\hline Parameter & Est. & Std. & HPD CI $(95 \%)$ \\
\hline Panel A: Gamma $(\gamma, 1)$ & & & \\
$\phi$ & 0.9691 & 0.0034 & $(0.9623,0.9755)$ \\
$\sigma$ & 0.1255 & 0.0073 & $(0.1104,0.1396)$ \\
$\gamma$ & 0.9487 & 0.0087 & $(0.9319,0.9658)$ \\
Panel B: Weibull $(v, 1)$ & & & \\
$\phi$ & 0.9732 & 0.0026 & $(0.9680,0.9784)$ \\
$\sigma$ & 0.1133 & 0.0054 & $(0.1024,0.1239)$ \\
$v$ & 0.9472 & 0.0050 & $(0.9372,0.9567)$ \\
& & & \\
Panel C: $\operatorname{Exp}(1)$ & & & \\
$\phi$ & 0.9661 & 0.0037 & $(0.9587,0.9730)$ \\
$\sigma$ & 0.1377 & 0.0077 & $(0.1230,0.1531)$ \\
\hline
\end{tabular}


Table 5: True and estimated parameters of the ASCD model based on the IBM duration data.

\begin{tabular}{cccc}
\hline Parameter & Est. & Std. & HPD CI $(95 \%)$ \\
\hline
\end{tabular}

Panel A: $\operatorname{Gamma}(\gamma, 1)$

$\begin{array}{lrrr}\phi & 0.9624 & 0.0048 & (0.9530,0.9715) \\ \psi & -0.0384 & 0.0091 & (-0.0561,-0.0207) \\ \sigma & 0.1400 & 0.0100 & (0.1207,0.1586) \\ \gamma & 0.9694 & 0.0105 & (0.9466,0.9874)\end{array}$

Panel B: Weibull $(v, 1)$

$\begin{array}{lrrr}\phi & 0.9724 & 0.0029 & (0.9666,0.9779) \\ \psi & -0.0401 & 0.0074 & (-0.0549,-0.0257) \\ \sigma & 0.1145 & 0.0060 & (0.1029,0.1258) \\ v & 0.9473 & 0.0058 & (0.9355,0.09578)\end{array}$

Panel C: $\operatorname{Exp}(1)$

\begin{tabular}{lrrr}
$\phi$ & 0.9558 & 0.0045 & $(0.9471,0.9645)$ \\
$\psi$ & -0.0521 & 0.0078 & $(-0.0673,-0.0368)$ \\
$\sigma$ & 0.1552 & 0.0085 & $(0.1390,0.1721)$ \\
\hline
\end{tabular}


To assess the goodness-of-fit of the ASCD models applied to the IBM duration data, we examine the PITs produced by the fitted models. Since the results are quite similar, below we only discuss the ASCD-W model. Figure 6 includes the scatter plot and the histogram of the PITs generated from the model, while Figure 7 plots the empirical CDF of the PITs with CDF of a uniform distribution over the interval $[0,1]$. The KS test rejects the null hypothesis that the PITs follow a uniform distribution over the interval $[0,1]$. We conclude that the fitted ASCD-W model does not seem to fit the IBM transaction data well. This finding is similar to that reported by Bauwens and Veredas (2004), where the authors commented that there is no generally accepted method that is suitable to check whether a latent variable model fits the data. In their paper, the authors checked the realized two innovations visually by using the $p$-value plots. They concluded that these plots do not confirm that the two innovations follow the distributions assumed for the SCD model. To explain the poor fit, the authors provided a plausible conjecture asserting that the durations may well be fractionally integrated or, perhaps, a more flexible model may be needed. One such a model is proposed by Jasiak (1998). Feng, Jiang, and Song (2004) also find that the transformed SCD model, with three types of distributions for the observation innovations, fits the right tails of the duration distributions very well, but not the left tail. Observing the second panel in Figure 6 and the CDF plots in Figure 7, we can see that the intensity of small durations is around value of 0.1 . This seems to indicate that small durations are not well described by the model, probably due to our assumption about the form of the distribution of the innovations in the observation equation.

Figure 8 compares the observed IBM duration time series with the insample and out-of-sample one-step-ahead forecast durations. The three time series of the forecast durations are similar and resemble the true duration dynamics. This indicates that the ASCD model is able to forecast the IBM durations reasonably well.

To choose a better SCD model for the data, we calculate the DIC values from the three fitted ASCD models and three SCD models without a correlation. The DIC measures are listed in Table 6. The smallest values is from the ASCD model with an Exponential innovation.

Table 7 compares the estimated parameters of the transformed SCD models with and without a leverage effect. In the table, columns titled SCD$\mathrm{MN}(2)-\mathrm{C}$ and SCD-MN(2)-L present estimates from the two-component mixture of the bivariate normal approach proposed in Xu, Knight, and Wirjanto 

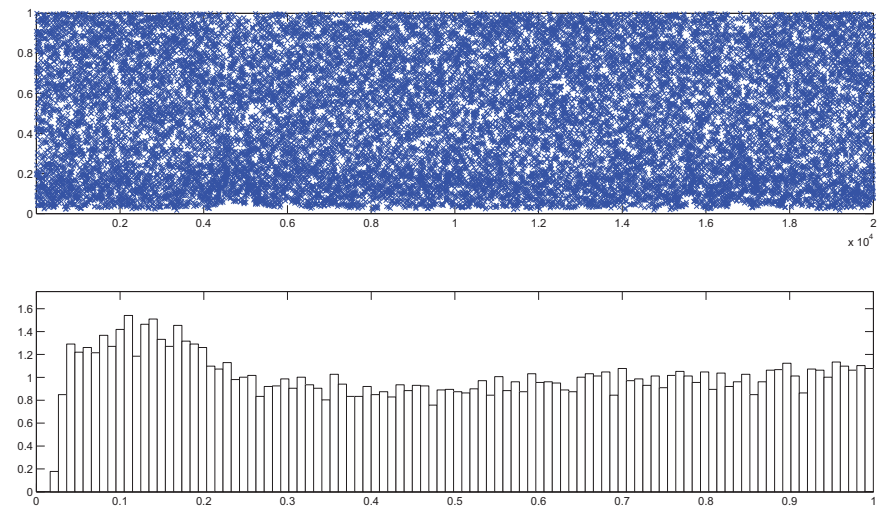

Figure 6: Analysis of the PITs from the ASCD-W model based on the IBM duration data. The top panel shows the scatter plot of $u(t)$ while the bottom the histogram of $u(t)$.

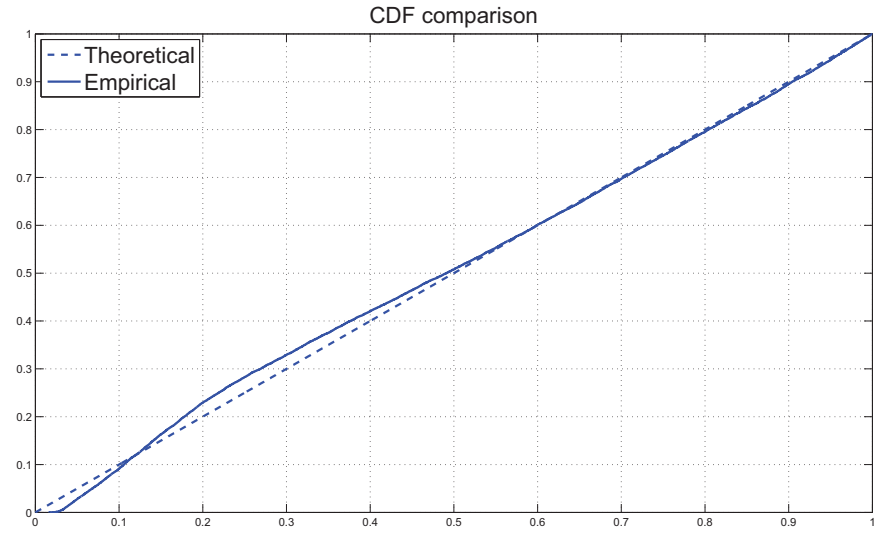

Figure 7: Comparison between the theoretical CDF of the uniform distribution over the interval $[0,1]$ and the empirical CDF of the PITs from the ASCD-W model based on the IBM duration data. 


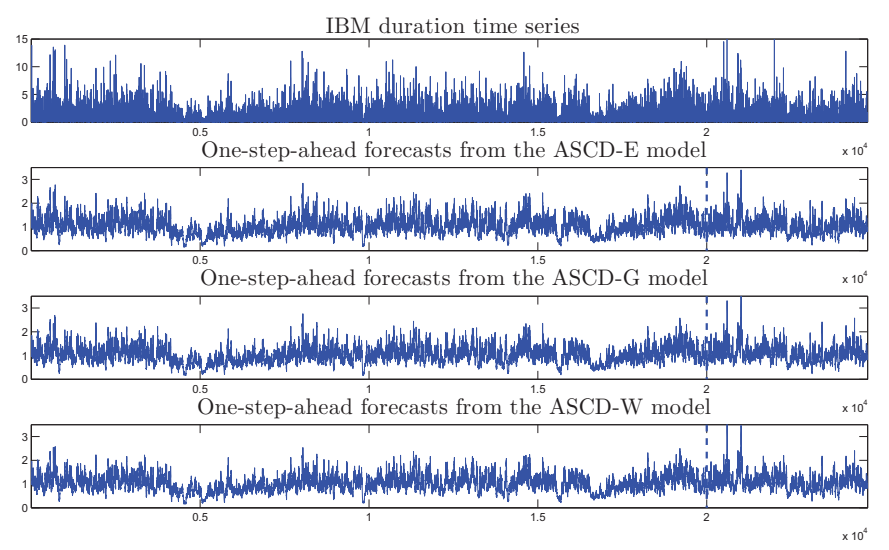

Figure 8: Comparison between the observed durations and the one-step-ahead forecasted durations of the IBM stock under the ASCD model with three innovation distribution assumptions.

Table 6: Model selection for the benchmark data of the IBM stock transactions.

\begin{tabular}{ccccccc}
\hline Criterion & $\begin{array}{c}\text { SCD } \\
(\psi=0)\end{array}$ & $\begin{array}{c}\text { ASCD-E } \\
(\psi \neq 0)\end{array}$ & $\begin{array}{c}\text { SCD } \\
(\psi=0)\end{array}$ & $\begin{array}{c}\text { ASCD-G } \\
(\psi \neq 0)\end{array}$ & $\begin{array}{c}\text { SCD } \\
(\psi=0)\end{array}$ & $\begin{array}{c}\text { SCD-W } \\
(\psi \neq 0)\end{array}$ \\
\hline & & & & & & \\
$\bar{D}$ & 35530.0 & 35413.7 & 35566.7 & 35449.4 & 35591.3 & 35475.4 \\
$P_{D}$ & -1335.3 & -1407.5 & -1266.0 & -1363.2 & -1194.7 & -1279.4 \\
DIC & 34194.8 & $\mathbf{3 4 0 0 6 . 2}$ & 34300.6 & 34086.3 & 34396.5 & 34195.9 \\
\hline
\end{tabular}


(2011) with contemporaneous and lagged intertemporal dependence structures, respectively. The column titled SCD-W includes the estimates from Knight and Ning (2008), whereas the last two columns provide the parameter estimates from Feng, Jiang, and Song (2004). Since the log transformation of the observation equation is one-to-one, this comparison is reasonable. In Table 7, the values of $\rho$ in the second and third columns are estimated correlations between the log-transformed innovations and the innovations of the latent processes, while the values of $\rho$ in the last two columns are the coefficients of the lagged intertemporal term in the latent $\mathrm{AR}(1)$ equation defined in Feng, Jiang, and Song (2004). We see that there is a positive "leverage effect" between the two innovations of the transformed duration model. In contrast, our models delivers a more interpretable leverage effect that captures the dependence structure between the IBM duration and the latent log duration processes.

Table 7: Estimated parameters of the SCD models from some competing methods based on the transformed IBM duration data.

\begin{tabular}{cccccc}
\hline Parameter & SCD-MN(2)-C & SCD-MN(2)-L & SCD-W & SCD-W-L & SCD-G-L \\
\hline & 0.9619 & 0.9490 & 0.9751 & 0.9716 & 0.9649 \\
$\sigma$ & 0.1371 & 0.1580 & 0.1157 & 0.1100 & 0.1293 \\
$v$ & n.a. & n.a. & 1.0445 & 0.9404 & n.a. \\
$\gamma$ & n.a. & n.a. & n.a. & n.a. & 0.9551 \\
$\rho$ & 0.0467 & 0.0598 & n.a. & 0.0125 & 0.0024 \\
\hline
\end{tabular}

\section{Conclusion}

To describe the dynamic of financial duration time series, we propose an ASCD model with a leverage effect. By introducing a correlation between two innovations, the leverage effect between the observed durations and their volatilities can be captured. In order to estimate the parameters and the latent states, we use the slice sampler within the MCMC method, which allows us to work with the data on the original scale. Another important advantage of our method is that the log expectations of the durations can be estimated as a by-product of the MCMC process. Our estimation method is flexible and allows us to conduct one-step-ahead forecasts in a straightforward manner. Simulation studies and an application of our method to the IBM transaction 
data demonstrate that the proposed ASCD model is able to capture different properties of financial duration series and to provide duration forecasts. By applying our method to the IBM transaction data, we have found a significantly negative correlation between the duration time series and the state variable.

\section{Appendix A. Technical proofs}

Proof of Proposition 1. From the latent AR(1) equation (10), we have

$$
\begin{aligned}
h_{t}= & \mu(1-\phi)+\phi \mu(1-\phi)+\phi^{2} \mu(1-\phi)+\ldots \\
& +\psi \epsilon_{t}+\psi \phi \epsilon_{t-1}+\psi \phi^{2} \epsilon_{t-2}+\ldots \\
& +\sigma \eta_{t}+\sigma \phi \eta_{t-1}+\sigma \phi^{2} \eta_{t-2}+\ldots
\end{aligned}
$$

Hence

$$
\begin{aligned}
\mu_{H} & =E\left(e^{h_{t}}\right) \\
& =e^{\mu+1 / 2 \sigma^{2} /\left(1-\phi^{2}\right)}\left[\prod_{i=0}^{\infty}\left(1-\psi \phi^{i}\right)\right]^{-\gamma}, \text { if }\left|\psi \phi^{i}\right|<1, i=1,2, \ldots
\end{aligned}
$$

Similarly, we have

$$
E\left(e^{2 h_{t}}\right)=e^{2 \mu+2 \sigma^{2} /\left(1-\phi^{2}\right)}\left[\prod_{i=0}^{\infty}\left(1-2 \psi \phi^{i}\right)\right]^{-\gamma}, \text { if }\left|\psi \phi^{i}\right|<1 / 2, i=1,2, \ldots
$$

Then the variance of $H_{t}$ is

$$
\begin{aligned}
\sigma_{H}^{2} & =e^{2 \mu+2 \sigma^{2} /\left(1-\phi^{2}\right)}\left[\prod_{i=0}^{\infty}\left(1-2 \psi \phi^{i}\right)\right]^{-\gamma}-e^{2 \mu+\sigma^{2} /\left(1-\phi^{2}\right)}\left[\prod_{i=0}^{\infty}\left(1-\psi \phi^{i}\right)\right]^{-2 \gamma} \\
& =e^{2 \mu+\sigma^{2} /\left(1-\phi^{2}\right)}\left\{e^{\sigma^{2} /\left(1-\phi^{2}\right)}\left[\prod_{i=0}^{\infty}\left(1-2 \psi \phi^{i}\right)\right]^{-\gamma}-\left[\prod_{i=0}^{\infty}\left(1-\psi \phi^{i}\right)\right]^{-2 \gamma}\right\} \\
& =\mu_{H}^{2}\left(e^{\sigma^{2} /\left(1-\phi^{2}\right)} \prod_{i=0}^{\infty} \frac{\left(1-\psi \phi^{i}\right)^{2 \gamma}}{\left(1-2 \psi \phi^{i}\right)^{\gamma}}-1\right), \text { if }\left|\psi \phi^{i}\right|<1 / 2, i=1,2, \ldots
\end{aligned}
$$

Now we calculate the mean and the variance of $y_{t}$.

$$
\begin{aligned}
E\left(y_{t}\right) & =E\left[\exp \left(h_{t}\right) \epsilon_{t}\right] \\
& =E\left[\exp \left(h_{t}\right)\right] E\left[\epsilon_{t}\right] \\
& =g_{1} \mu_{H} .
\end{aligned}
$$


Since

$$
\begin{aligned}
E\left(y_{t}^{2}\right) & =E\left[\exp \left(2 h_{t}\right) \epsilon_{t}^{2}\right] \\
& =E\left[\exp \left(2 h_{t}\right)\right] E\left[\epsilon_{t}^{2}\right]
\end{aligned}
$$

then we have

$$
\begin{aligned}
\operatorname{Var}\left(y_{t}\right) & =E\left(y_{t}^{2}\right)-\left[E\left(y_{t}\right)\right]^{2} \\
& =e^{2 \mu+2 \sigma^{2} /\left(1-\phi^{2}\right)}\left[\prod_{i=0}^{\infty}\left(1-2 \psi \phi^{i}\right)\right]^{-\gamma} g_{2}-g_{1}^{2} e^{2 \mu+\sigma^{2} /\left(1-\phi^{2}\right)}\left[\prod_{i=0}^{\infty}\left(1-\psi \phi^{i}\right)\right]^{-2 \gamma} \\
& =\mu_{y}^{2}\left(\kappa e^{\sigma^{2} /\left(1-\phi^{2}\right)} \prod_{i=0}^{\infty} \frac{\left(1-\psi \phi^{i}\right)^{2 \gamma}}{\left(1-2 \psi \phi^{i}\right)^{\gamma}}-1\right), \text { if }\left|\psi \phi^{i}\right|<1 / 2, i=1,2, \ldots
\end{aligned}
$$

Proof of Proposition 3. The stationarity of the process $\left\{d_{t}\right\}$ can be easily checked. Based on Equation (10), when $|\phi|<1$ holds, we have that

$$
h_{t}=\mu+\psi \sum_{k=0}^{\infty} \phi^{k} \epsilon_{t-k}+\sigma \sum_{k=0}^{\infty} \phi^{k} \eta_{t-k}
$$

Hence, we have

$$
d_{t}=e_{t}+\mu+\psi \sum_{k=0}^{\infty} \phi^{k} \epsilon_{t-k}+\sigma \sum_{k=0}^{\infty} \phi^{k} \eta_{t-k}
$$

It is easy to see that $\left\{d_{t}\right\}$ is the sum of two weakly stationary $\operatorname{AR}(\infty)$ processes; therefore, $\left\{d_{t}\right\}$ is weakly stationary.

Let $X_{t}^{\prime}=\left(d_{t}, e_{t}, \epsilon_{t}\right)_{1 \times 3}, V_{t}^{\prime}=\left(e_{t}, \epsilon_{t}, \eta_{t+1}\right)_{1 \times 3}$,

$$
A=\left(\begin{array}{ccc}
\phi & -\psi & \psi \\
0 & 0 & 0 \\
0 & 0 & 0
\end{array}\right)_{3 \times 3} \quad \text { and } \quad \Omega=\left(\begin{array}{ccc}
1 & \sigma & 0 \\
1 & 0 & 0 \\
0 & 0 & 1
\end{array}\right)_{3 \times 3}
$$

Equations (12) and (10), which formulate the transformed SCD model, can be written as

$$
X_{t}=A X_{t-1}+\Omega V_{t}
$$


To show that $X_{t}$ is geometrically ergodic, we first show that the Markov chain $X_{t}$ is irreducible and aperiodic. Notice that the generalized controllability matrix is

$$
C=\left(\begin{array}{lll}
1 & \sigma & 0 \\
1 & 0 & 0 \\
0 & 0 & 1
\end{array}\right)
$$

which is a full-rank matrix. Therefore, the chain $X_{t}$ is forward accessible based on the Proposition 7.1.4 in Meyn and Tweedie (1993). Moreover, it is obvious that $X^{*}=(0,0,0)$ is a global attracting state, so the chain is irreducible and aperiodic according to Theorem 7.2.6 in Meyn and Tweedie (1993).

Now, we show that the chain $X_{t}$ is geometrically ergodic. Let the test function be $U\left(x_{t}\right)=\|\Sigma x\|+1$, where $\|\Sigma\|$ is a specially chosen matrix for some $\epsilon>0$, and the test set $C=\left\{x \in R^{3}: U(x) \leq c\right.$ for some $\left.c<\infty\right\}$, where $\|$.$\| denote the Euclidean norm for a vector or the spectral norm for a$ matrix.

Then, we have

$$
E\left[U\left(x_{t}\right) \mid x_{t-1}=x\right] \leq(1-\epsilon) U(x)+\delta I_{\epsilon}(x)
$$

for some $\delta<\infty$ and for all $x$, where $I_{\epsilon_{t}}(x)$ is an indicator function defined as usual. From Theorem 15.0.1 in Gouriérous (1993), we have that $X_{t}$ is geometrically ergodic, and so are $d_{t}$.

Since the log transformation is one-to-one, conditions for ergodicity and stationarity for $\left\{y_{t}\right\}$ are the same as for $\left\{d_{t}\right\}$. 


\section{Appendix B. Full conditionals of parameters and latent states in the ASCD model}

-The full conditional of $\mu$, given a normal prior $\mathcal{N}\left(\alpha_{\mu}, \beta_{\mu}^{2}\right)$, is expressed as

$$
\begin{aligned}
& f\left(\mu \mid \mathbf{y}, \mu, \phi, \psi, \sigma^{2}\right) \\
& \propto f(\mathbf{h} \mid \theta, \mathbf{y}) \exp \left\{-\frac{\left(\mu-\alpha_{\mu}\right)^{2}}{2 \beta_{\mu}^{2}}\right\} \\
& \propto f\left(h_{1} \mid \theta\right) \prod_{t=1}^{T-1} f\left(h_{t+1} \mid h_{t}, \theta, y_{t}\right) \exp \left\{-\frac{\left(\mu-\alpha_{\mu}\right)^{2}}{2 \beta_{\mu}^{2}}\right\} \\
& \propto \exp \left\{-\frac{\sum_{t=1}^{T-1}\left[\left(h_{t+1}-\mu\right)-\phi\left(h_{t}-\mu\right)-\psi \exp \left(-h_{t}\right) y_{t}\right]^{2}}{2 \sigma^{2}}\right\} \\
& \quad \times \exp \left\{-\frac{\left(1-\phi^{2}\right)\left(h_{1}-\mu\right)^{2}}{2 \sigma^{2}}\right\} \exp \left\{-\frac{\left(\mu-\alpha_{\mu}\right)^{2}}{2 \beta_{\mu}^{2}}\right\} \\
& =\exp \left(-\frac{1}{2}\left[\mu^{2}\left\{\frac{1-\phi^{2}}{\sigma^{2}}+\frac{(T-1)(1-\phi)^{2}}{\sigma^{2}}+\frac{1}{\beta_{\mu}^{2}}\right\}\right.\right. \\
& \left.\left.\quad-2 \mu\left\{\frac{h_{1}\left(1-\phi^{2}\right)}{\sigma^{2}}+\frac{(1-\phi) \sum_{t=1}^{T-1}\left(h_{t+1}-\phi h_{t}-\psi \exp \left(-h_{t}\right) y_{t}\right)}{\sigma^{2}}+\frac{\alpha_{\mu}}{\beta_{\mu}^{2}}\right\}\right]\right) \\
& \propto \mathcal{N}\left(\frac{b}{a}, \frac{1}{a}\right) .
\end{aligned}
$$

where

$$
\begin{aligned}
& a=\frac{1-\phi^{2}}{\sigma^{2}}+\frac{(T-1)(1-\phi)^{2}}{\sigma^{2}}+\frac{1}{\beta_{\mu}^{2}}, \\
& b=\frac{h_{1}\left(1-\phi^{2}\right)}{\sigma^{2}}+\frac{(1-\phi) \sum_{t=1}^{T-1}\left(h_{t+1}-\phi h_{t}-\psi \exp \left(-h_{t}\right) y_{t}\right)}{\sigma^{2}}+\frac{\alpha_{\mu}}{\beta_{\mu}^{2}} .
\end{aligned}
$$

- The full conditional of $\phi$, given a normal prior $\mathcal{N}\left(\alpha_{\phi}, \beta_{\phi}^{2}\right)$, is the follow- 
ing

$$
\begin{aligned}
& f\left(\phi \mid \mathbf{y}, \mu, \psi, \sigma^{2}\right) \\
& \propto f(\mathbf{h} \mid \theta, \mathbf{y}) \exp \left\{-\frac{\left(\phi-\alpha_{\phi}\right)^{2}}{2 \beta_{\phi}^{2}}\right\} \\
& \propto f\left(h_{1} \mid \theta\right) \prod_{t=1}^{T-1} f\left(h_{t+1} \mid h_{t}, \theta, y_{t}\right) \exp \left\{-\frac{\left(\phi-\alpha_{\phi}\right)^{2}}{2 \beta_{\phi}^{2}}\right\} \\
& \propto \exp \left\{-\frac{\sum_{t=1}^{T-1}\left[\left(h_{t+1}-\mu\right)-\phi\left(h_{t}-\mu\right)-\psi \exp \left(-h_{t}\right) y_{t}\right]^{2}}{2 \sigma^{2}}\right\} \\
& \quad \times \exp \left\{-\frac{\left(1-\phi^{2}\right)\left(h_{1}-\mu\right)^{2}}{2 \sigma^{2}}\right\} \exp \left\{-\frac{\left(\phi-\alpha_{\phi}\right)^{2}}{2 \beta_{\phi}^{2}}\right\}\left(1-\phi^{2}\right)^{\frac{1}{2}} \\
& =\exp \left(-\frac{1}{2}\left[\phi^{2}\left\{\frac{-\left(h_{1}-\mu\right)^{2}}{\sigma^{2}}+\frac{\sum_{t=1}^{T-1}\left(h_{t}-\mu\right)^{2}}{\sigma^{2}}+\frac{1}{\beta_{\phi}^{2}}\right\}\right.\right. \\
& \left.\left.\quad-2 \phi\left\{\frac{\sum_{t=1}^{T-1}\left(h_{t}-\mu\right)\left(h_{t+1}-\mu-\psi \exp \left(-h_{t}\right) y_{t}\right)}{\sigma^{2}}+\frac{\alpha_{\phi}}{\beta_{\phi}^{2}}\right\}\right]\right)\left(1-\phi^{2}\right)^{\frac{1}{2}} \\
& \propto \mathcal{N}\left(\frac{d}{c}, \frac{1}{c}\right)\left(1-\phi^{2}\right)^{\frac{1}{2}},
\end{aligned}
$$

where

$$
\begin{aligned}
& c=\frac{-\left(h_{1}-\mu\right)^{2}}{\sigma^{2}}+\frac{\sum_{t=1}^{T-1}\left(h_{t}-\mu\right)^{2}}{\sigma^{2}}+\frac{1}{\beta_{\phi}^{2}}, \\
& d=\frac{\sum_{t=1}^{T-1}\left(h_{t}-\mu\right)\left(h_{t+1}-\mu-\psi \exp \left(-h_{t}\right) y_{t}\right)}{\sigma^{2}}+\frac{\alpha_{\phi}}{\beta_{\phi}^{2}} .
\end{aligned}
$$


- The full conditional of $\psi$, given a normal prior density $\mathcal{N}\left(\alpha_{\psi}, \beta_{\psi}^{2}\right)$, is

$$
\begin{aligned}
& f\left(\psi \mid \mathbf{y}, \mu, \phi, \sigma^{2}\right) \\
& \propto f(\mathbf{h} \mid \theta, \mathbf{y}) \exp \left\{-\frac{\left(\psi-\alpha_{\psi}\right)^{2}}{2 \beta_{\psi}^{2}}\right\} \\
& \propto f\left(h_{1} \mid \theta\right) \prod_{t=1}^{T-1} f\left(h_{t+1} \mid h_{t}, \theta, y_{t}\right) \exp \left\{-\frac{\left(\psi-\alpha_{\psi}\right)^{2}}{2 \beta_{\psi}^{2}}\right\} \\
& \propto \exp \left\{-\frac{\sum_{t=1}^{T-1}\left[\left(h_{t+1}-\mu\right)-\phi\left(h_{t}-\mu\right)-\psi \exp \left(-h_{t}\right) y_{t}\right]^{2}}{2 \sigma^{2}}\right\} \\
& \quad \times \exp \left\{-\frac{\left(\psi-\alpha_{\psi}\right)^{2}}{2 \beta_{\psi}^{2}}\right\} \\
& =\exp \left(-\frac{1}{2}\left[\psi^{2}\left\{\frac{\sum_{t=1}^{T-1}\left[\exp \left(-h_{t}\right) y_{t}\right]^{2}}{\sigma^{2}}+\frac{1}{\beta_{\psi}^{2}}\right\}\right.\right. \\
& \left.\left.\quad-2 \psi\left\{\frac{\sum_{t=1}^{T-1}\left[\exp \left(-h_{t}\right) y_{t}\left\{\left(h_{t+1}-\mu\right)-\phi\left(h_{t}-\mu\right)\right\}\right]}{\sigma^{2}}+\frac{\alpha_{\psi}}{\beta_{\psi}^{2}}\right\}\right]\right) \\
& \propto \mathcal{N}\left(\frac{b_{1}}{a_{1}}, \frac{1}{a_{1}}\right),
\end{aligned}
$$

where

$$
\begin{aligned}
a_{1} & =\frac{\sum_{t=1}^{T-1}\left[\exp \left(-h_{t}\right) y_{t}\right]^{2}}{\sigma^{2}}+\frac{1}{\beta_{\psi}^{2}}, \\
b_{1} & =\frac{\sum_{t=1}^{T-1}\left[\exp \left(-h_{t}\right) y_{t}\left\{\left(h_{t+1}-\mu\right)-\phi\left(h_{t}-\mu\right)\right\}\right]}{\sigma^{2}}+\frac{\alpha_{\psi}}{\beta_{\psi}^{2}} .
\end{aligned}
$$

- The full conditional of $\sigma^{2}$, given an inverse Gamma prior density $f\left(\alpha_{\sigma^{2}}, \beta_{\sigma^{2}}\right)$, 
is the following

$$
\begin{aligned}
& f\left(\sigma^{2} \mid \mathbf{y}, \mu, \psi, \phi\right) \\
& \propto f(\mathbf{h} \mid \theta, \mathbf{y}) f\left(\alpha_{\sigma^{2}}, \beta_{\sigma^{2}}\right) \\
& \propto f\left(h_{1} \mid \theta\right) \prod_{t=1}^{T-1} f\left(h_{t+1} \mid h_{t}, \theta, y_{t}\right) f\left(\alpha_{\sigma^{2}}, \beta_{\sigma^{2}}\right) \\
& \propto \exp \left\{-\frac{\sum_{t=1}^{T-1}\left[\left(h_{t+1}-\mu\right)-\phi\left(h_{t}-\mu\right)-\psi \exp \left(-h_{t}\right) y_{t}\right]^{2}}{2 \sigma^{2}}\right\} \\
& \quad \times \exp \left\{-\frac{\left(1-\phi^{2}\right)\left(h_{1}-\mu\right)^{2}}{2 \sigma^{2}}\right\} \times\left(\frac{1}{\sigma^{2}}\right)^{\frac{T}{2}} \frac{\left(\beta_{\sigma^{2}}\right)^{\alpha_{\sigma^{2}}} e^{-\beta_{\sigma^{2}} / \sigma_{\sigma^{2}}}}{\Gamma\left(\alpha_{\sigma^{2}}\right)\left(\sigma_{\sigma^{2}}\right)^{\alpha_{\sigma^{2}}+1}} \\
& \propto \exp \left(\frac{\beta_{\sigma}^{2}+\frac{1}{2}\left(h_{1}-\mu\right)^{2}\left(1-\phi^{2}\right)+\frac{1}{2} \sum_{t=1}^{T-1}\left[\left(h_{t+1}-\mu\right)-\phi\left(h_{t}-\mu\right)-\psi \exp \left(-h_{t}\right) y_{t}\right]^{2}}{\sigma^{2}}\right\} \\
& \quad \times\left(\frac{1}{\sigma^{2}}\right)^{\left(\alpha_{\sigma^{2}}+\frac{T}{2}\right)+1} \\
& \propto \mathcal{I} G(a, b),
\end{aligned}
$$

which is an inverse Gamma distribution with parameters,

$$
\begin{aligned}
& a=\alpha_{\sigma^{2}}+\frac{T}{2}, \\
& b=\beta_{\sigma^{2}}+\frac{1}{2}\left(h_{1}-\mu\right)^{2}\left(1-\phi^{2}\right)+\frac{1}{2} \sum_{t=1}^{T-1}\left[\left(h_{t+1}-\mu\right)-\phi\left(h_{t}-\mu\right)-\psi \exp \left(-h_{t}\right) y_{t}\right]^{2} .
\end{aligned}
$$

- Full conditional of $h_{1}$

$$
\begin{aligned}
& P\left(h_{1} \mid y_{1: T}, h_{2}, \theta\right) \\
& =c_{1} f\left(y_{1} \mid h_{1}\right) f\left(h_{1} \mid \theta\right) f\left(h_{1} \mid h_{2}, y_{1}, \theta\right) \\
& =c_{2} f\left(y_{1} \mid h_{1}\right) \exp \left\{-\frac{\left(1-\phi^{2}\right)\left(h_{1}-\mu\right)^{2}}{2 \sigma^{2}}\right\} \\
& \quad \times \exp \left\{-\frac{\left[\left(h_{2}-\mu\right)-\phi\left(h_{1}-\mu\right)-\psi \exp \left(-h_{1}\right) y_{1}\right]^{2}}{2 \sigma^{2}}\right\} \\
& <c_{2} f\left(y_{1} \mid h_{1}\right) \exp \left\{-\frac{\left(1-\phi^{2}\right)\left(h_{1}-\mu\right)^{2}}{2 \sigma^{2}}\right\},
\end{aligned}
$$

where $c_{1}$ and $c_{2}$ are the two normalizing constants. 
- Full conditional of $h_{T}$

$$
\begin{aligned}
& f\left(h_{T} \mid y_{1: T}, h_{T-1}, \theta\right) \\
& \propto f\left(y_{T} \mid h_{T}\right) f\left(h_{T} \mid h_{T-1}, y_{T-1}, \theta\right) \\
& \propto f\left(y_{T} \mid h_{T}\right) \times \exp \left\{-\frac{\left[\left(h_{T}-\mu\right)-\phi\left(h_{T-1}-\mu\right)-\psi \exp \left(-h_{T-1}\right) y_{T-1}\right]^{2}}{2 \sigma^{2}}\right\} .
\end{aligned}
$$

\section{References}

Akaike, H. 1987. Factor Analysis and AIC. Psychometrika 52: 317-332.

Bauwens, L., and F. Galli. 2009. Efficient Important Sampling for ML Estimation of SCD Models. Computational Statistics and Data Analysis 53: 1974-1992.

Bauwens, L., and M. Lubrano. 1998. Bayesian Inference on GARCH Models Using the Gibbs Sampler. Econometrics Journal 1: C23-C26.

Bauwens, L., and D. Veredas. 2004. The Stochastic Conditional Duration Model: A Latent Variable Model for the Analysis of Financial Durations. Journal of Econometrics 119(2): 381-482.

Berg, A., R. Meyer, and J. Yu. 2004. Deviance Information Criterion for Comparing Stochastic Volatility Models. Journal of Business and Economic Statistics 22: 107-120.

Bollerslev, T. 1986. Generalized Autoregressive Conditional Heteroskedasticity. Journal of Econometrics 31: 307-327.

Chib, S., F. Nardari, and N. Shephard. 2006. Analysis of High Dimensional Multivariate Stochastic Volatility Models. Journal of Econometrics 134: 341-371.

Diebold, F. X., T. A. Guther, and A. S. Tay. 1998. Evaluating Density Forecasts with Applications to Financial Risk Management. International Economic Review 39: 863-883.

Durbin, J., and S. Koopman. 2004. Monte Carlo Maximum Likelihood Estimation for Non-Gaussian State Space Models. Biometrika 84: 669-684. 
Engle, R. F. 1982. Autoregressive Conditional Heteroskedasticity with Estimates of the Variance of United Kingdom Inflation. Econometrica 50(4): 987-1007.

Engle, R. F., and J. R. Russell. 1998. Autoregressive Conditional Duration: A New Approach for Irregularly Spaced Transaction Data. Econometrica 19: 69-90.

Edwards, R. G. and A. D. Sokal. 1988. Generalization of the FortuinKasteleyn-Swendsen-Wang Representation and Monte Carlo Algorithm. Phys. Rev. D 38(6): 2009-2012.

Feng, D., G. J. Jiang, and P. X. K. Song. 2004. Stochastic Conditional Duration Models with Leverage Effect for Financial Transaction Data. Journal of Financial Econometrics 2(3): 390-421.

Geweke, J. 1993. Bayesian Treatment of the Independent Student-t Linear Model. Journal of Applied Econometrics 8: S19-S40.

Gouriérous, C. 1997. "ARCH Models and Financial Application." New York: Springer-Verlag.

Jacquier, E., N. G. Polson, and P. E. Rossi. 2004. Bayesian Analysis of Stochastic Volatility Models with Fat-tails and Correlated Errors. Journal of Econometrics 122: 185-212.

Jasiak, J. 1998. Persistence in Intertrade Durations. Finance 19: 166-195.

Knight, J. L., and C. Ning. 2008. Estimation of the Stochastic Conditional Duration Model via Alternative Methods. Econometrics Journal 11(3): 573-592.

Meyn, S. P. and R. L. Tweedie. 1993. "Markov Chain and Stochastic Stability". New York: Springer-Verlag.

Mira, A., and L. Tierney. 2002. Efficiency and Convergence Properties of Slice Samplers. Scandinavian Journal of Statistics 29: 1-12.

Neal, R. N. 2003. Slice Sampling. The Annals of Statistics 31: 705-767. 
Pitt, M., and N. Shephard. 1999a. Time Varying Convariances: A Factor Stochastic Volatility Approach. In: Bernardo, J. M., Berger, J. O., David, A. P., Smith, A. F. M., eds. Bayesian Statistics 6: 547-570. Oxford University Press.

Pitt, M., and N. Shephard. 1999b. Filtering via Simulation: Auxiliary Particle Filters. Journal of the American Statistical Association 94: 590-599.

Roberts G. O., and J. S. Rosenthal. 1999. Convergence of Slice Sampler Markov Chains. Journal of the Royal Statistical Society. Series B (Statistical Methodology) 61(3): 643-660.

Sandmann G., and S. J. Koopman. 1998. Estimation of Stochastic Volatility Models via Monte Carlo Maximum Likelihood. Journal of Econometrics 87: 271-301.

Schwarz G. 1978. Estimating the Dimension of a Model. Annals of Statistics 6(2): 461-464.

Spiegelhalter, D. J., N. G. Best, B. P. Carlin, and A. van der Linde. 2002. Bayesian Measures of Model Complexity and Fit (with discussion). Journal of the Royal Statistical Society, Ser. B 64: 583-639.

Strickland, C. M., C. S. Forbes, and G. M. Martin. 2006. Bayesian Analysis of the Stochastic Conditional Duration Model. Computational Statistics and Data Analysis 50: 2247-2267.

Taylor, S. J. 1986. Modeling Financial Time Series. Chichester, Uk:Wiley.

Xu, D., J. Knight, and T. S. Wirjanto. 2011. Asymmetric Stochastic Conditional Duration Model-A Mixture-of-Normal Approach. Journal of Financial Econometrics 9(3): 469-488.

Yu, J. 2005. On Leverage in a Stochastic Volatility Model. Journal of Econometrics 127: 165-178.

Zhang, X., and L. King. 2008. Box-Cox Stochastic Volatility Models with Heavy-tails and Correlated Errors. Journal of Empirical Finance 15: 549566. 